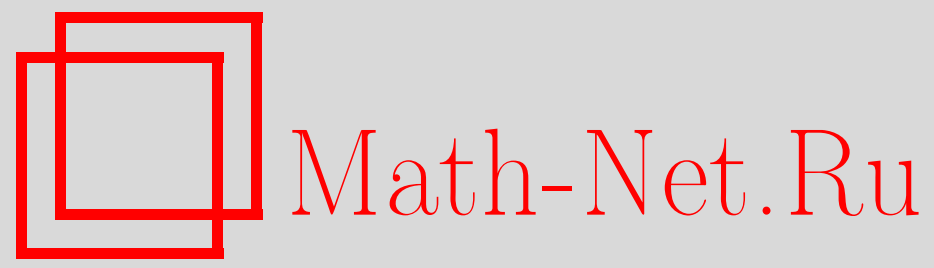

Ю. В. Шеретов, О классах точных решений квазигидродинамической системы, Вестник ТвГУ. Серия: Прикладная математика, 2020, выпуск 2, 5-17

DOI: https://doi.org/10.26456/vtpmk592

Использование Общероссийского математического портала Math-Net.Ru подразумевает, что вы прочитали и согласны с пользовательским соглашением

http://www.mathnet.ru/rus/agreement

Параметры загрузки:

IP : 3.93 .64 .190

26 апреля 2023 г., $17: 38: 45$ 


\section{МАТЕМАТИЧЕСКОЕ МОДЕЛИРОВАНИЕ, ЧИСЛЕННЫЕ МЕТОДЫ И КОМПЛЕКСЫ ПРОГРАММ}

УДК $517.95,532.5$

\section{О КЛАССАХ ТОЧНЫХ РЕШЕНИЙ КВАЗИГИДРОДИНАМИЧЕСКОЙ СИСТЕМЫ}

Шеретов Ю.В.

Тверской государственный университет, г. Тверь

Поступила в редакчию 10.06.2020, после переработки 18.06.2020.

Получено условие совпадения решений уравнений Навье-Стокса и квазигидродинамической системы. Показано, что многие известные решения системы Навье-Стокса подчиняются этому условию. Рассмотрены классы точных решений, общих для уравнений Навье-Стокса и квазигидродинамической системы. Применение принципа суперпозиции при построении точных решений показано на конкретных примерах, как в стационарном, так и в нестационарном случае.

Ключевые слова: система Навье-Стокса, квазигидродинамическая система, условие совпадения решений, классы точных решений, принцип суперпозиции.

Вестник ТвГУ. Серия: Прикладная математика. 2020. № 2. С. 5-17. https://doi.org/10.26456/vtpmk592

\section{Введение}

Построению точных решений классической системы Навье-Стокса в динамике вязкой несжимаемой жидкости посвящена обширная научная литература [1-5]. Эти решения играют важную роль в численном анализе, так как используются в качестве тестов компьютерных программ. Альтернативная квазигидродинамическая (КГД) система была предложена в 1993 году автором [6]. Обоснованию подхода, выявлению связей с классической теорией Навье-Стокса посвящены монографии $[7,8]$. Анализ точных решений КГД системы был продолжен в работах [9-13]. В частности, в [10] для построения стационарных решений, общих для систем Навье-Стокса и КГД, использовался принцип суперпозиции. В цилиндрических координатах этот принцип применялся в [9]. Идея расщепления уравнений Навье-Стокса, фактически приводящая к принципу суперпозиции, рассматривалась в [14].

В настоящей работе рассмотрены классы точных решений квазигидродинамической системы, удовлетворяющих предложенному автором условию. Все построенные решения являются точными и для соответствующей системы Навье-Стокса. Указанному условию подчиняются многие классические решения уравнений Навье-Стокса. 


\section{1. Условие совпадения решений уравнений Навье-Стокса и квазигидро- динамической системы}

Квазигидродинамическая система для слабосжимаемой вязкой жидкости без учета внешних сил в стандартных обозначениях может быть представлена в виде

$$
\begin{gathered}
\operatorname{div} \vec{u}=\operatorname{div} \vec{w}, \\
\frac{\partial \vec{u}}{\partial t}+((\vec{u}-\vec{w}) \cdot \nabla) \vec{u}+\nabla p=\nu \Delta \vec{u}+\nu \nabla(\operatorname{div} \vec{u})+\operatorname{div}(\vec{u} \otimes \vec{w}) .
\end{gathered}
$$

Вектор $\vec{w}$ вычисляется по формуле

$$
\vec{w}=\tau((\vec{u} \cdot \nabla) \vec{u}+\nabla p) .
$$

Символом $\nu$ обозначен коэффициент кинематической вязкости жидкости, $\Delta-$ оператор Лапласа, действующий на векторное поле. Постоянная средняя плотность жидкости $\rho$ положена равной единице. Система (1.1) - (1.2) замкнута относительно неизвестных функций - скорости $\vec{u}=\vec{u}(\vec{x}, t)$ и давления $p=p(\vec{x}, t)$. Характерное время релаксации $\tau$ вычисляется по формуле

$$
\tau=\frac{\nu}{c_{s}^{2}},
$$

где $c_{s}$ - скорость звука в жидкости. Параметры $\nu$ и $\tau$ являются положительными константами.

Пренебрегая в системе (1.1) - (1.2) членами, содержащими $\tau$, получим классическую систему Навье-Стокса в динамике вязкой несжимаемой жидкости:

$$
\begin{gathered}
\operatorname{div} \vec{u}=0, \\
\frac{\partial \vec{u}}{\partial t}+(\vec{u} \cdot \nabla) \vec{u}+\nabla p=\nu \Delta \vec{u} .
\end{gathered}
$$

Будем рассматривать гладкие (бесконечно-дифференцируемые) решения систем Навье-Стокса и КГД. Справедлива

Теорема 1. Пусть пара бесконечно-дифференцируемых функиий $(\vec{u}, p)$ образует точное решение системы Навъе-Стокса (1.4) - (1.5) и выполнено условие

$$
(\vec{w} \cdot \nabla) \vec{u}+(\vec{u} \cdot \nabla) \vec{w}=0 .
$$

Тогда $(\vec{u}, p)$ является гладким решением квазигидродинамической системы.

Доказательство. Пусть $(\vec{u}, p)$ - бесконечно-дифференцируемое решение системы Навье-Стокса (1.4) - (1.5). Тогда выполняется равенство (1.4). Подействуем оператором «div» на обе части равенства (1.5). Принимая во внимание (1.3) и (1.4), получим

$$
\operatorname{div} \vec{w}=0 .
$$

В силу (1.4), (1.7) первое уравнение квазигидродинамической системы обращается в истинное равенство. Преобразуем (1.2) к эквивалентному виду

$$
\frac{\partial \vec{u}}{\partial t}+(\vec{u} \cdot \nabla) \vec{u}+\nabla p-\nu \Delta \vec{u}=
$$




$$
=\nu \nabla(\operatorname{div} \vec{u})+\vec{w} \operatorname{div} \vec{u}+(\vec{w} \cdot \nabla) \vec{u}+(\vec{u} \cdot \nabla) \vec{w} .
$$

Учтем (1.4) и (1.5). При выполнении условия (1.6) пара функций $(\vec{u}, p)$ обращает (1.8) в истинное равенство.

Замечание 1. Условие (1.6) было получено автором.

См., например, [8], с. 98.

Замечание 2. Условие (1.6) может быть представлено в эквивалентном виде

$$
\nabla(\vec{u} \cdot \vec{w})-\vec{u} \times \operatorname{rot} \vec{w}-\vec{w} \times \operatorname{rot} \vec{u}=0 .
$$

Для доказательства достаточно воспользоваться известным векторным тождеством, приведенным в [1] на с. 32.

\section{2. Общие решения системы Навье-Стокса и квазигидродинамической системы в декартовых координатах}

Запишем систему Навье-Стокса (1.4) - (1.5) в декартовых координатах:

$$
\begin{gathered}
\frac{\partial u_{x}}{\partial x}+\frac{\partial u_{y}}{\partial y}+\frac{\partial u_{z}}{\partial z}=0 \\
\frac{\partial u_{x}}{\partial t}+u_{x} \frac{\partial u_{x}}{\partial x}+u_{y} \frac{\partial u_{x}}{\partial y}+u_{z} \frac{\partial u_{x}}{\partial z}+\frac{\partial p}{\partial x}=\nu\left(\frac{\partial^{2} u_{x}}{\partial x^{2}}+\frac{\partial^{2} u_{x}}{\partial y^{2}}+\frac{\partial^{2} u_{x}}{\partial z^{2}}\right) \\
\frac{\partial u_{y}}{\partial t}+u_{x} \frac{\partial u_{y}}{\partial x}+u_{y} \frac{\partial u_{y}}{\partial y}+u_{z} \frac{\partial u_{y}}{\partial z}+\frac{\partial p}{\partial y}=\nu\left(\frac{\partial^{2} u_{y}}{\partial x^{2}}+\frac{\partial^{2} u_{y}}{\partial y^{2}}+\frac{\partial^{2} u_{y}}{\partial z^{2}}\right) \\
\frac{\partial u_{z}}{\partial t}+u_{x} \frac{\partial u_{z}}{\partial x}+u_{y} \frac{\partial u_{z}}{\partial y}+u_{z} \frac{\partial u_{z}}{\partial z}+\frac{\partial p}{\partial z}=\nu\left(\frac{\partial^{2} u_{z}}{\partial x^{2}}+\frac{\partial^{2} u_{z}}{\partial y^{2}}+\frac{\partial^{2} u_{z}}{\partial z^{2}}\right)
\end{gathered}
$$

Она замкнута относительно неизвестных функций - компонент вектора скорости $u_{x}=u_{x}(x, y, z, t), u_{y}=u_{y}(x, y, z, t), u_{z}=u_{z}(x, y, z, t)$ и давления $p=p(x, y, z, t)$. Займемся поиском классов точных решений уравнений Навье-Стокса (2.1) - (2.4), подчиняющихся условию (1.6). По теореме, доказанной в предыдущем пункте, найденные решения будут также удовлетворять КГД системе (1.1) - (1.2), выписанной в декартовых координатах.

Класс 1. Будем искать решение системы (2.1) - (2.4) в виде

$$
\begin{gathered}
u_{x}=0, \quad u_{y}=0, \quad u_{z}=u_{z}(x, y, t), \\
p=A(t) z+B(t) .
\end{gathered}
$$

Здесь $A(t)$ и $B(t)$ - неизвестные функции времени. Для зависимостей (2.5), (2.6) декартовы компоненты вектора $\vec{w}$ выглядят следующим образом:

$$
w_{x}=0, \quad w_{y}=0, \quad w_{z}=\tau A(t) .
$$


Условие (1.6) принимает вид

$$
\tau A(t) \frac{\partial}{\partial z}\left(\begin{array}{c}
0 \\
0 \\
u_{z}(x, y, t)
\end{array}\right)+u_{z}(x, y, t) \frac{\partial}{\partial z}\left(\begin{array}{c}
0 \\
0 \\
\tau A(t)
\end{array}\right)=0 .
$$

Равенство (2.8), очевидно, выполняется. Подстановка $(2,5),(2.6)$ в (2.1) - (2.3) приводит к истинным равенствам. Соотношение (2.4) принимает вид

$$
\frac{\partial u_{z}}{\partial t}=\nu\left(\frac{\partial^{2} u_{z}}{\partial x^{2}}+\frac{\partial^{2} u_{z}}{\partial y^{2}}\right)-A(t) .
$$

Линейное двумерное уравнение теплопроводности (2.9) с источниковым членом $(-A(t))$ в правой части хорошо изучено. Можно привести множество точных решений этого уравнения для конкретных задач. Ограничимся несколькими известными примерами.

Пример 1. Задача Стокса. Пусть жидкость занимает полупространство $x \geqslant 0$, а плоскость уоz, являющаяся твердой границей, совершает гармонические колебания параллельно оси $o z$ с положительной частотой $\omega$. Решение системы (2.1) (2.4), отвечающее этой задаче, будем искать в виде

$$
\begin{gathered}
u_{x}=0, \quad u_{y}=0, \quad u_{z}=u_{z}(x, t), \\
p=p_{0} .
\end{gathered}
$$

Из (2.9) находим одномерное линейное нестационарное уравнение теплопроводности

$$
\frac{\partial u_{z}}{\partial t}=\nu \frac{\partial^{2} u_{z}}{\partial x^{2}},
$$

которое имеет решение (см. [2], с. 122-123) типа затухающей бегущей волны

$$
u_{z}=U_{0} \exp \left(-\frac{x}{\delta}\right) \cos \left(\frac{x}{\delta}-\omega t\right) .
$$

Здесь $\delta=\sqrt{2 \nu / \omega}, U_{0}$ - заданная положительная постоянная.

Пример 2. Рассмотрим течение жидкости между двумя параллельными плоскостями с координатами $x=0$ и $x=H>0$. Левая плоскость совершает гармонические колебания в направлении оси $o z$ с положительной частотой $\omega$. Скорость на ней меняется по закону

$$
\left.u_{z}\right|_{x=0}=U_{0} \cos (\omega t) .
$$

Правая плоскость неподвижна. Для системы Навье-Стокса эта задача решена в [2] на с. 128. Схема такая же, как и в предыдущем примере. Зависимость $u_{z}=u_{z}(x, t)$ имеет вид

$$
u_{z}=\operatorname{Re}\left(U_{0} e^{-i \omega t} \frac{\sin (k(H-x))}{\sin (k H)}\right) .
$$

Здесь $i$ - мнимая единица, $k=(1+i) \sqrt{\omega / 2 \nu}$. Символом $R e$ обозначена действительная часть комплексного числа. 
Класс 2. Займемся поиском решений системы (2.1) - (2.4) вида

$$
\begin{gathered}
u_{x}=0, \quad u_{y}=u_{y}(x, t), \quad u_{z}=u_{z}(x, t), \\
p=A(t) y+B(t) z+C(t) .
\end{gathered}
$$

Здесь $A(t), B(t)$ и $C(t)$ - неизвестные функции времени. Для распределений скорости (2.16) и давления (2.17) декартовы компоненты вектора $\vec{w}$ выглядят так:

$$
w_{x}=0, \quad w_{y}=\tau A(t), \quad w_{z}=\tau B(t) .
$$

Условие (1.6) запишется следующим образом:

$$
\tau\left(A(t) \frac{\partial}{\partial y}+B(t) \frac{\partial}{\partial z}\right)\left(\begin{array}{c}
0 \\
u_{y}(x, t) \\
u_{z}(x, t)
\end{array}\right)+\left(u_{y} \frac{\partial}{\partial y}+u_{z} \frac{\partial}{\partial z}\right)\left(\begin{array}{c}
0 \\
\tau A(t) \\
\tau B(t)
\end{array}\right)=0
$$

Соотношение (2.19) представляет собой истинное равенство. Для зависимостей (2.16) и (2.17) равенства (2.1) и (2.2) удовлетворяются тождественно. Уравнения (2.3), (2.4) принимают, соответственно, вид

$$
\begin{aligned}
\frac{\partial u_{y}}{\partial t} & =\nu \frac{\partial^{2} u_{y}}{\partial x^{2}}-A(t), \\
\frac{\partial u_{z}}{\partial t} & =\nu \frac{\partial^{2} u_{z}}{\partial x^{2}}-B(t) .
\end{aligned}
$$

Проблема свелась к анализу двух независимых линейных одномерных нестационарных уравнений теплопроводности с источниковыми членами. Это позволяет применять принцип суперпозиции при построении точных решений уравнений Навье-Стокса и КГД. Продемонстрируем этот принцип лишь на одном примере.

Пример 3. Рассмотрим движение жидкости между двумя параллельными плоскими пластинами, которые расположены перпендикулярно оси ox и имеют координаты $x=0$ и $x=H>0$, соответственно. Правая пластина колеблется с положительной частотой $\omega_{1}$ параллельно оси оу. Компоненты скорости на ней равны

$$
\left.u_{y}\right|_{x=H}=U_{0} \cos \left(\omega_{1} t\right),\left.\quad u_{z}\right|_{x=H}=0
$$

Левая пластина совершает гармонические колебания параллельно оси $о z$ с положительной частотой $\omega_{2}$. Граничные условия для составляющих поля скорости на ней выглядят следующим образом:

$$
\left.u_{y}\right|_{x=0}=0,\left.\quad u_{z}\right|_{x=0}=V_{0} \cos \left(\omega_{2} t\right) .
$$

В $(2.22)$ и (2.23) символами $U_{0}$ и $V_{0}$ обозначены заданные положительные константы. Давление в жидкости считаем постоянным:

$$
p=p_{0}=\text { const }>0 .
$$


Тогда $A(t)=0, B(t)=0$. Уравнения $(2.20)$ и (2.21) принимают вид

$$
\begin{aligned}
& \frac{\partial u_{y}}{\partial t}=\nu \frac{\partial^{2} u_{y}}{\partial x^{2}} \\
& \frac{\partial u_{z}}{\partial t}=\nu \frac{\partial^{2} u_{z}}{\partial x^{2}} .
\end{aligned}
$$

Решения выписанных краевых задач находятся по стандартной схеме (см. [2], с. 128):

$$
\begin{gathered}
u_{y}=\operatorname{Re}\left(U_{0} e^{-i \omega_{1} t} \frac{\sin \left(k_{1} x\right)}{\sin \left(k_{1} H\right)}\right), \\
u_{z}=\operatorname{Re}\left(V_{0} e^{-i \omega_{2} t} \frac{\sin \left(k_{2}(H-x)\right)}{\sin \left(k_{2} H\right)}\right) .
\end{gathered}
$$

Здесь $k_{1}=(1+i) \sqrt{\omega_{1} / 2 \nu}, k_{2}=(1+i) \sqrt{\omega_{2} / 2 \nu}$. Составляющая скорости $u_{x}$ равна нулю.

\section{3. Общие решения системы Навье-Стокса и квазигидродинамической системы в цилиндрических координатах}

Классическая система Навье-Стокса для вязкой несжимаемой жидкости в цилиндрических координатах [2] выглядит так:

$$
\begin{gathered}
\frac{1}{r} \frac{\partial\left(r u_{r}\right)}{\partial r}+\frac{1}{r} \frac{\partial u_{\varphi}}{\partial \varphi}+\frac{\partial u_{z}}{\partial z}=0 \\
\frac{\partial u_{r}}{\partial t}+u_{r} \frac{\partial u_{r}}{\partial r}+\frac{u_{\varphi}}{r} \frac{\partial u_{r}}{\partial \varphi}+u_{z} \frac{\partial u_{r}}{\partial z}-\frac{u_{\varphi}^{2}}{r}+\frac{\partial p}{\partial r}= \\
=\nu\left[\frac{1}{r} \frac{\partial}{\partial r}\left(r \frac{\partial u_{r}}{\partial r}\right)+\frac{1}{r^{2}} \frac{\partial^{2} u_{r}}{\partial \varphi^{2}}+\frac{\partial^{2} u_{r}}{\partial z^{2}}-\frac{u_{r}}{r^{2}}-\frac{2}{r^{2}} \frac{\partial u_{\varphi}}{\partial \varphi}\right] \\
\frac{\partial u_{\varphi}}{\partial t}+u_{r} \frac{\partial u_{\varphi}}{\partial r}+\frac{u_{\varphi}}{r} \frac{\partial u_{\varphi}}{\partial \varphi}+u_{z} \frac{\partial u_{\varphi}}{\partial z}+\frac{u_{r} u_{\varphi}}{r}+\frac{1}{r} \frac{\partial p}{\partial \varphi}= \\
\left.=\frac{1}{r} \frac{\partial}{\partial r}\left(r \frac{\partial u_{\varphi}}{\partial r}\right)+\frac{1}{r^{2}} \frac{\partial^{2} u_{\varphi}}{\partial \varphi^{2}}+\frac{\partial^{2} u_{\varphi}}{\partial z^{2}}-\frac{u_{\varphi}}{r^{2}}+\frac{2}{r^{2}} \frac{\partial u_{r}}{\partial \varphi}\right] \\
\frac{\partial u_{z}}{\partial t}+u_{r} \frac{\partial u_{z}}{\partial r}+\frac{u_{\varphi}}{r} \frac{\partial u_{z}}{\partial \varphi}+u_{z} \frac{\partial u_{z}}{\partial z}+\frac{\partial p}{\partial z}= \\
=\nu\left[\frac{1}{r} \frac{\partial}{\partial r}\left(r \frac{\partial u_{z}}{\partial r}\right)+\frac{1}{r^{2}} \frac{\partial^{2} u_{z}}{\partial \varphi^{2}}+\frac{\partial^{2} u_{z}}{\partial z^{2}}\right]
\end{gathered}
$$

Неизвестными в ней являются компоненты вектора скорости $u_{r}=u_{r}(r, \varphi, z, t)$, $u_{\varphi}=u_{\varphi}(r, \varphi, z, t), u_{z}=u_{z}(r, \varphi, z, t)$ в базисе $\left(\vec{e}_{r}, \vec{e}_{\varphi}, \vec{e}_{z}\right)$ и давление $p=p(r, \varphi, z, t)$. 
Класс 3. Будем искать решение системы (3.1) - (3.4) в виде

$$
\begin{gathered}
u_{r}=0, \quad u_{\varphi}=u_{\varphi}(r, t), \quad u_{z}=u_{z}(r, t), \\
p=\widehat{p}(r, t)+A(t) z+B(t) .
\end{gathered}
$$

Для зависимостей $(3.5),(3.6)$ составляющие вектора $\vec{w}$ в базисе $\left(\vec{e}_{r}, \vec{e}_{\varphi}, \vec{e}_{z}\right)$ таковы:

$$
w_{r}=\tau\left(\frac{\partial \widehat{p}}{\partial r}-\frac{u_{\varphi}^{2}}{r}\right), \quad w_{\varphi}=0, \quad w_{z}=\tau A(t) .
$$

Если

$$
\frac{\partial \widehat{p}}{\partial r}=\frac{u_{\varphi}^{2}}{r},
$$

то условие (1.6) выполняется. Принимая во внимание (3.8), подставим (3.5) и (3.6) в (3.1) - (3.4). Это приводит к соотношениям

$$
\begin{gathered}
\frac{\partial u_{\varphi}}{\partial t}=\nu\left[\frac{1}{r} \frac{\partial}{\partial r}\left(r \frac{\partial u_{\varphi}}{\partial r}\right)-\frac{u_{\varphi}}{r^{2}}\right], \\
\frac{\partial u_{z}}{\partial t}=\frac{\nu}{r} \frac{\partial}{\partial r}\left(r \frac{\partial u_{z}}{\partial r}\right)-A(t) .
\end{gathered}
$$

Система, эквивалентная (3.8) - (3.10), была выписана в [14] на с. 167. Идея расщепления системы Навье-Стокса состоит в том, что уравнения (3.9) и (3.10) могут быть решены по отдельности, независимо друг от друга. Функция $\widehat{p}=\widehat{p}(r, t)$ находится из (3.8) простым интегрированием:

$$
\widehat{p}(r, t)=p_{0}+\int_{r_{0}}^{r} \frac{u_{\varphi}^{2}\left(r_{*}, t\right)}{r_{*}} d r_{*} .
$$

Здесь $r$ и $r_{0}$ - заданные неотрицательные числа, $p_{0}$ - положительное число.

Пример 4. Задача Озина. В формулах (3.5), (3.6) положим $u_{z}=0, A(t)=0$, $B(t)=0$. Тогда (3.10) удовлетворяется тождественно. Уравнение (3.9) имеет частное решение

$$
u_{\varphi}=\frac{\Gamma}{2 \pi r}\left[1-\exp \left(-\frac{r^{2}}{4 \nu t}\right)\right] .
$$

Распределение давления может быть найдено по формуле

$$
p=p_{\infty}-\int_{r}^{+\infty} \frac{u_{\varphi}^{2}\left(r_{*}, t\right)}{r_{*}} d r_{*} .
$$

Символом Г обозначена положительная константа, $p_{\infty}-$ значение давления в бесконечно удаленной точке. Решение (3.12) - (3.13) описывает эволюцию изолированной вихревой нити в вязкой жидкости.

Пример 5. Суперпозиция течений Куэтта между соосными цилиндрами. Рассмотрим стационарное течение жидкости, заключенной между двумя вращающимися с постоянными угловыми скоростями $\Omega_{1}$ и $\Omega_{2}$ соосными цилиндрами. Пусть 
$R_{1}$ и $R_{2}$ - их радиусы, причем $R_{2}>R_{1}>0$. Выберем цилиндрические координаты $r, \varphi, z$ с осью $о z$ по оси цилиндров. Предположим еще, что внутренний цилиндр радиуса $R_{1}$ движется вдоль оси oz с постоянной скоростью $U$, а составляющая скорости вдоль оси оz внешнего цилиндра радиуса $R_{2}$ равна нулю. Решение будем искать в виде

$$
\begin{gathered}
u_{r}=0, \quad u_{\varphi}=u_{\varphi}(r), \quad u_{z}=u_{z}(r), \\
p=p(r) .
\end{gathered}
$$

Зависимости (3.14) и (3.15) есть частный случай (3.5) и (3.6) для установившихся течений. Градиент давления в направлении оси оz отсутствует. Уравнения (3.9) и (3.10) принимают вид

$$
\begin{gathered}
\frac{\partial}{\partial r}\left(\frac{1}{r} \frac{\partial\left(r u_{\varphi}\right)}{\partial r}\right)=0, \\
\frac{1}{r} \frac{\partial}{\partial r}\left(r \frac{\partial u_{z}}{\partial r}\right)=0 .
\end{gathered}
$$

С учетом граничных условий получаем

$$
\begin{gathered}
u_{\varphi}=\frac{\Omega_{2} R_{2}^{2}-\Omega_{1} R_{1}^{2}}{R_{2}^{2}-R_{1}^{2}} r+\frac{\left(\Omega_{1}-\Omega_{2}\right) R_{1}^{2} R_{2}^{2}}{R_{2}^{2}-R_{1}^{2}} \frac{1}{r}, \\
u_{z}=U \frac{\ln \left(r / R_{2}\right)}{\ln \left(R_{1} / R_{2}\right)}, \quad R_{1} \leqslant r \leqslant R_{2} .
\end{gathered}
$$

Компонента скорости $u_{r}$ равна нулю. Распределение давления имеет вид

$$
\begin{aligned}
& p(r)=p_{1}+\frac{1}{\left(R_{2}^{2}-R_{1}^{2}\right)^{2}}\left[\left(\Omega_{2} R_{2}^{2}-\Omega_{1} R_{1}^{2}\right)^{2} \frac{r^{2}-R_{1}^{2}}{2}+\right. \\
& \quad+2\left(\Omega_{2} R_{2}^{2}-\Omega_{1} R_{1}^{2}\right)\left(\Omega_{1}-\Omega_{2}\right) R_{1}^{2} R_{2}^{2} \ln \left(\frac{r}{R_{1}}\right)+ \\
& \left.+\left(\Omega_{1}-\Omega_{2}\right)^{2} R_{1}^{4} R_{2}^{4}\left(\frac{1}{2 R_{1}^{2}}-\frac{1}{2 r^{2}}\right)\right], \quad R_{1} \leqslant r \leqslant R_{2} .
\end{aligned}
$$

Здесь $p_{1}=p\left(R_{1}\right)$.

\section{4. Потенциальные и однородно-винтовые течения}

Итак, предложенному автором условию

$$
(\vec{w} \cdot \nabla) \vec{u}+(\vec{u} \cdot \nabla) \vec{w}=0
$$

подчиняются многие известные решения классической системы Навье-Стокса, как в стационарном, так и в нестационарном случае. Это условие может быть представлено в эквивалентном виде

$$
(((\vec{u} \cdot \nabla) \vec{u}+\nabla p) \cdot \nabla) \vec{u}+(\vec{u} \cdot \nabla)((\vec{u} \cdot \nabla) \vec{u}+\nabla p)=0 .
$$

Все построенные решения удовлетворяют также квазигидродинамической системе. Выписанному условию подчиняются стационарные потенциальные течения жидкости и нестационарные однородно-винтовые течения. 
Действительно, пусть в некоторой области $V$ пространства $\mathbb{R}_{\vec{x}}^{3}$ задана гармоническая функция $\Phi=\Phi(\vec{x})$. Определим поле скорости по формуле

$$
\vec{u}=\nabla \Phi .
$$

Тогда

$$
\operatorname{div} \vec{u}=0, \quad \operatorname{rot} \vec{u}=0, \quad \Delta \vec{u}=0 .
$$

Давление определим по формуле Бернулли

$$
p=p_{0}-\frac{\vec{u}^{2}}{2} .
$$

Здесь $p_{0}=$ const. Нетрудно проверить [8], что пара функций $(\vec{u}, p)$, определяемых формулами (4.3) и (4.5), является общим точным решением стационарных систем Эйлера, Навье-Стокса и КГД. Представим вектор $\vec{w}$ в виде

$$
\vec{w}=\tau\left(\nabla\left(\frac{\vec{u}^{2}}{2}+p\right)+[\operatorname{rot} \vec{u} \times \vec{u}]\right) .
$$

Из (4.4) и (4.5) следует, что $\vec{w}=0$. Таким образом, условие (4.1) для установившихся потенциальных течений жидкости выполняется.

Пусть нестационарное движение жидкости является однородно-винтовым. Это означает, что существует такая постоянная $\lambda \neq 0$, что выполнено соотношение

$$
\operatorname{rot} \vec{u}=\lambda \vec{u} \text {. }
$$

Пусть пара функций $(\vec{u}, p)$, где

$$
p=p_{0}(t)-\frac{\vec{u}^{2}}{2}
$$

$p_{0}(t)$ - произвольная функция времени, задает однородно-винтовое решение системы Навье-Стокса. Подстановка (4.7) и (4.8) в (4.6) дает $\vec{w}=0$. В силу доказанной теоремы $(\vec{u}, p)$ является также однородно-винтовым решением квазигидродинамической системы.

\section{Заключение}

Точные решения квазигидродинамической системы, не починяющиеся условию (4.1), существуют. Пример приведен в [7] на с. 107. Расширение класса точных решений КГД системы, не удовлетворяющих уравнениям Навье-Стокса, является актуальной и трудной задачей. Будут ли они поточечно или равномерно стремиться к соответствующему решению системы Навье-Стокса при $\tau \rightarrow+0$ ? Ответов на эти вопросы пока нет.

Предложенная автором квазигидродинамическая система применялась при компьютерных расчетах многих практически важных задач гидродинамики. Ограничимся ссылкой на одну из последних работ [15], где рассмотрена проблема моделирования дискового насоса в пакете OpenFOAM. Эффективные насосы, способные поддерживать кровообращение в сердце человека, широко используются в современной медицине. 


\section{Список литературы}

[1] Лойцянский Л.Г. Механика жидкости и газа. М.: Наука, 1987. 840 с.

[2] Ландау Л.Д., Лифшиц Е.М. Гидродинамика. М.: Наука, 1986. 736 с.

[3] Riley N., Drazin P.G. The Navier-Stokes equations: A classification of flows and exact solutions. Cambridge: Cambridge University Press, 2006. 196 p.

[4] Пухначев В.В. Симметрии в уравнениях Навье-Стокса // Успехи механики. 2006. № 1. C. 6-76.

[5] Wang C.Y. Exact solutions of the unsteady Navier-Stokes equations // Applied Mechanics Reviews. 1989. Vol. 42, № 11. Part 2. Pp. S269-S282.

[6] Шеретов Ю.В. О единственности решений одной диссипативной системы гидродинамического типа // Математическое моделирование. 1994. Т. 6, № 10. C. $35-45$.

[7] Шеретов Ю.В. Динамика сплошных сред при пространственно-временном осреднении. М., Ижевск: НИЦ «Регулярная и хаотическая динамика», 2009. $400 \mathrm{c}$.

[8] Шеретов Ю.В. Регуляризованные уравнения гидродинамики. Тверь: Тверской государственный университет, 2016. 222 с.

[9] Шеретов Ю.В. О точных решениях стационарных квазигидродинамических уравнений в цилиндрических координатах // Вестник ТвГУ. Серия: Прикладная математика. 2017. № 1. С. 85-94.

[10] Шеретов Ю.В. Об общих точных решениях стационарной системы НавьеСтокса и квазигидродинамической системы, не удовлетворяющих уравнениям Эйлера // Вестник ТвГУ. Серия: Прикладная математика. 2017. № 2. С. 5-15. https://doi.org/10.26456/vtpmk169

[11] Шеретов Ю.В. Об общих точных решениях системы Навье-Стокса и квазигидродинамической системы для нестационарных течений // Вестник ТвГУ. Серия: Прикладная математика. 2017. № 3. C. 13-25. https://doi.org/10.26456/ vtpmk 176

[12] Шеретов Ю.В. Точные решения квазигидродинамической системы на основе формулы Био-Савара // Вестник ТвГУ. Серия: Прикладная математика. 2019. № 1. C. 38-49. https://doi.org/10.26456/vtpmk525

[13] Шеретов Ю.В. О решениях задачи Коши для квазигидродинамической системы // Вестник ТвГУ. Серия: Прикладная математика. 2020. № 1. С. 84-96.

[14] Сизых Г.Б. Расщепление уравнений Навье-Стокса для одного класса осесимметричных течений // Вестник Самарского государственного технического университета. Серия: Физико-математические науки. 2020. Т. 24, № 1. С. 163173. https://doi.org/10.14498/vsgtu1740 
[15] Stenina T., Elizarova T., Ryazanov D., Ryabinkin E. Implementation of regularized equations for the disk pump simulation problem in OpenFOAM // Proceedings of 2019 Ivannikov ISPRAS Open Conference, ISPRAS. 2019. Pp. 124130.

\section{Образец цитирования}

Шеретов Ю.В. О классах точных решений квазигидродинамической системы // Вестник ТвГУ. Серия: Прикладная математика. 2020. №2. С. 5-17. https://doi.org/10.26456/vtpmk592

\section{Сведения об авторах}

\section{1. Шеретов Юрий Владимирович}

заведующий кафедрой математического анализа Тверского государственного университета.

Россил, 170100, г. Тверь, ул. Желлбова, д. З3, ТвГУ.

E-mail: Sheretov.YV@tversu.ru 


\title{
ON CLASSES OF EXACT SOLUTIONS \\ OF QUASI-HYDRODYNAMIC SYSTEM
}

\author{
Sheretov Yurii Vladimirovich \\ Head of Mathematical Analysis department, Tver State University \\ Russia, 170100, Tver, Zhelyabova str., 33, TverSU. \\ E-mail: Sheretov.YV@tversu.ru
}

\begin{abstract}
Received 10.06.2020, revised 18.06.2020.
The condition for the coincidence of the solutions of Navier-Stokes equations and the quasi-hydrodynamic system is obtained. It is shown that many well-known solutions of the Navier-Stokes system obey this condition. Classes of exact solutions common to the Navier-Stokes equations and the quasi-hydrodynamic system are considered. The application of the principle of superposition to the constructing exact solutions is shown on concrete examples, both in the stationary and non-stationary cases.
\end{abstract}

Keywords: Navier-Stokes system, quasi-hydrodynamic system, condition for coincidence of solutions, classes exact solutions, principle of superposition.

\section{Citation}

Sheretov Yu.V., "On classes of exact solutions of quasi-hydrodynamic system", Vestnik TvGU. Seriya: Prikladnaya Matematika [Herald of Tver State University. Series: Applied Mathematics], 2020, № 2, 5-17 (in Russian). https://doi.org/10.26456/vtpmk592

\section{References}

[1] Lojtsyanskij L.G., Mekhanika zhidkosti i gaza [Fluid and Gas Mechanics], Nauka Publ., Moscow, 1987 (in Russian), 840 pp.

[2] Landau L.D., Lifshits E.M., Gidrodinamika [Hydrodynamics], Nauka Publ., Moscow, 1986 (in Russian), 736 pp.

[3] Riley N., Drazin P.G., The Navier-Stokes equations: A classification of flows and exact solutions, Cambridge University Press, Cambridge, 2006, 196 pp.

[4] Pukhnachev V.V., "Symmetries in the Navier-Stokes equations", Uspekhi mekhaniki [Achievements in Mechanics], 2006, № 1, 6-76 (in Russian).

[5] Wang C.Y., "Exact solutions of the unsteady Navier-Stokes equations", Applied Mechanics Reviews, 42:11, Part 2 (1989), S269-S282.

[6] Sheretov Yu.V., "On uniqueness of the solutions for one dissipative system of hydrodynamic type", Matematicheskoe modelirovanie [Mathematical Modeling], 6:10 (1994), 35-45 (in Russian). 
[7] Sheretov Yu.V., Dinamika sploshnykh sred pri prostranstvenno-vremennom osrednenii [Continuum Dynamics under Spatiotemporal Averaging], Regular and Chaotic Dynamics Publ., Moscow, Izhevsk, 2009 (in Russian), 400 pp.

[8] Sheretov Yu.V., Regulyarizovannye uravneniya gidrodinamiki [Regularized Hydrodynamic Equations], Tver State University, Tver, 2016 (in Russian), 222 pp.

[9] Sheretov Yu.V., "On the exact solutions of stationary quasi-hydrodynamic equations in cylindrical coordinates", Vestnik TvGU. Seriya: Prikladnaya Matematika [Herald of Tver State University. Series: Applied Mathematics], 2017, № 1, 85-94 (in Russian).

[10] Sheretov Yu.V., "On the common exact solutions of stationary Navier-Stokes and quasi-hydrodynamic systems, not satisfying to Euler equations", Vestnik TvGU. Seriya: Prikladnaya Matematika [Herald of Tver State University. Series: Applied Mathematics], 2017, № 2, 5-15 (in Russian), https://doi.org/10.26456/vtpmk169.

[11] Sheretov Yu.V., "On common exact solutions of Navier-Stokes and quasi-hydrodynamic systems for nonstationary flows", Vestnik TvGU. Seriya: Prikladnaya Matematika [Herald of Tver State University. Series: Applied Mathematics], 2017, № 3, 13-25 (in Russian), https://doi.org/10.26456/vtpmk176.

[12] Sheretov Yu.V., "Exact solutions of quasi-hydrodynamic system on the base of Biot-Savart formula", Vestnik TvGU. Seriya: Prikladnaya Matematika [Herald of Tver State University. Series: Applied Mathematics], 2019, № 1, 38-49 (in Russian), https://doi.org/10.26456/vtpmk525.

[13] Sheretov Yu.V., "On the solutions of Cauchy problem for quasi-hydrodynamic system", Vestnik TvGU. Seriya: Prikladnaya Matematika [Herald of Tver State University. Series: Applied Mathematics], 2020, № 1, 84-96 (in Russian).

[14] Sizykh G.B., "The splitting of Navier-Stokes equations for a class of axisymmetric flows", Vestnik Samarskogo Gosudarstvennogo Tekhnicheskogo Universiteta. Seriya Fiziko-Matematicheskie Nauki [Journal of Samara State Technical University, Ser. Physical and Mathematical Sciences], 24:1 (2020), 163-173 (in Russian), https://doi.org/10.14498/vsgtu1740.

[15] Stenina T., Elizarova T., Ryazanov D., Ryabinkin E., "Implementation of regularized equations for the disk pump simulation problem in OpenFOAM", Proceedings of 2019 Ivannikov ISPRAS Open Conference, ISPRAS, 2019, 124-130. 